\title{
Evaluating the Operational Performance and Financial Effects of a Drug Prior Authorization Program
}

Charles R. Phillips and Lon N. Larson

\section{OBJECTIVE:}

To present a managerial approach for evaluating the performance of a drug prior authorization (PA) program, illustrated through an evaluation of the lowa Medicaid drug PA program.

\section{DESIGN:}

A before-and-after comparison without a control group.

\section{SETTING:}

State of lowa Medicaid Program.

\section{PATIENTS:}

lowa Medicaid enrollees (approximately $250,000)$.

\section{INTERVENTION:}

Medicaid Drug Prior Authorization (PA) Program.

\begin{abstract}
MAIN OUTCOME MEASURES:
Operational and economic performance, including volume of prior authorization requests, PA program response times, PA approval rates by therapeutic category, medication utilization, and PA program savings and administrative costs.
\end{abstract}

\section{RESULTS:}

Overall, $82.9 \%$ of new and extension PA requests were approved for coverage. Of the new PA requests, $74.4 \%$ were approved for coverage, while $92.5 \%$ of PA extensions were approved. The average response time for new requests and extensions was 52.4 (s.d.=101.8) minutes. New PA responses averaged 73.2 (s.d.=119.6) minutes, while extensions averaged 28 (s.d.=7.5) minutes. The total net savings for antiarthritics, benzodiazepines, antiulcer, and antihistamines was $\$ 2.51$ million to $\$ 3.83$ million.

\section{CONCLUSION:}

The results are unique in providing both operational and economic performance of a drug PA program. The results show response times well below the HCFA mandated guidelines with relatively high approval rates. The economic results also suggest a large savings due to drug prior authorization. The evaluation procedures provide a workable method for program administrators to evaluate their own programs. In addition, the results provide a comparison for program administrators to judge performance.

\section{KEY WORDS:}

Drug costs, Drug utilization, Económics, Insurance, Pharmaceutical services, Medicaid cost analysis, Prior authorization

J Managed Care Pharm 1997; 3: 699-706
Prior authorization (PA) programs have become a staple in the management of prescription drug benefit programs. Used mainly to control the use of high-dollar prescription drugs, 42 state medical assistance programs have adopted PA programs.' As they have gained popularity, however, these programs have come under scrutiny by various groups ranging from consumers seeking fewer limitations on access and providers seeking fewer restrictions on practice to manufacturers seeking more open markets. Because of this, program administrators are continually developing and refining ways of measuring program savings, costs, and quality to justify their use.
The major determinants of whether these and other managed care principles are retained by program administrators revolve around cost savings, quality of care, and ease of administration. Although published findings are scarce, Smalley et al. studied the effect of a PA program on the Tennessee Medicaid population., ${ }^{2,3}$ They evaluated nonsteroidal antiinflammatory drugs (NSAIDs) and attempted to quantify both direct and indirect savings for the entire state Medicaid program. They estimated that the program saved $\$ 12.8$ million over a wo-year period with no concomitant increase in other Medicaid medical expenditures.

Authors

CHARLES R. PHILLIPS, PH.D., is an Assistant Professor and LON N. LARSON, PH.D., is a Professor at the Drake University, College of Pharmacy and Health Sciences, Des Moines, IA.

AUthor Correspondence: Charles R. Phillips, Ph.D., Drake University, College of Pharmacy and Health Sciences, 2507 University, Des Moines, LA 50311.

ACKNOWLEDGEMENT: The authors would like to acknowledge the contributions made by Joel Hanson, Randy Brentnall, and Debbie Moore in collecting and organizing data. At the time of the study, they were employed by the prior authorization programis fiscal agent, Unisys Corporation. The authors also would like to acknowledge the assistance of Joe Mahrenholz, R.Ph., M.S., and the lowa Deparment of Human Services. 
Table 1. Average Contact by Day of the Week and Type of Contact

\begin{tabular}{|c|c|c|c|}
\hline Day & $\begin{array}{c}\text { Average Number of New PA } \\
\text { and Extension Requests }\end{array}$ & $\begin{array}{c}\text { Average Number of } \\
\text { Other Contacts }\end{array}$ & $\begin{array}{c}\text { Average Number of } \\
\text { Total Contacts }\end{array}$ \\
\hline Monday & 98.0 & 49.5 & 147.5 \\
\hline Tuesday & 109.0 & 46.0 & 155.0 \\
\hline Wednesday & 75.5 & 37.0 & 1.12 .5 \\
\hline Thursday & 87.0 & 49.0 & 136.0 \\
\hline Friday & 93.0 & 33.0 & 126.0 \\
\hline Saturday* & 7.5 & 4.5 & 12.0 \\
\hline Sunday** & 3.0 & 1.0 & 4.0 \\
\hline
\end{tabular}

* Five hour day ** Four hour day

Similarly, Kotzan et al. evaluated the impact of the Georgia Medicaid PA program for NSAIDs. ${ }^{4}$ Following 80,064 continually-enrolled recipients receiving one or more NSAID agents throughout 1989 and the first seven months of 1990, the authors determined that total costs for NSAID therapy decreased by more than $\$ 3$ million in 1990 . They projected over $\$ 7$ million in savings for the year if all Georgia Medicaid recipients were included. This was associated with slightly higher costs for nonnarcotic analgesics. Overall, this study found the PA program was successful in containing drug costs while avoiding increased utilization of medical and physician services.

This article presents a managerial approach to evaluate and monitor the performance of a drug prior authorization program, illustrated through an evaluation of the lowa Medicaid drug PA program. The evaluation encompasses methods for measuring operational performance and estimating the effects of a PA program on drug use and expenditures. The focus is on cost savings and administrative performance. The perspective of the analysis is that of the third-party payor. Costs and administrative duties outside the realm of the program administrator, such as the time and effort spent by providers on PA requests, are beyond the scope of this analysis. All data required in the analysis are available from the drug plan administrator (or fiscal agent in Medicaid programs). While the PA program highlighted in this article is a public-sector program, the methods are equally applicable to private programs.

\section{PRIOR AUTHORIZATION}

The purpose of drug prior authorization is to affect prescribing patterns. More specifically, it is designed to limit or restrict selected high-cost prescribing decisions. For instance, PA guidelines may: 1) encourage the use of generics; 2) encourage the use of alternative, less costly drugs which can be used for the same conditions; 3) enforce step-therapy (i.e., initiate therapy with drug A; if no success, then try drug B); 4) restrict the situations in which a high-cost drug may be prescribed (e.g., the patient must meet certain clinical criteria before the drug can be used); or 5) encourage alternative (lower) doses of the prescribed product.

The four components that help determine a PA program's financial impact are: 1) the immediate effect of the PA denials; 2) the effect of PA on prescribing patterns; 3 ) the effect of PA decisions on the use of other services; and 4) the cost of implementing and running the PA program itself. The first and last components are the most directly associated with the program and the easiest to measure.

As PA requests are denied, savings can be accumulated. For instance, if a prescription costing $\$ 50$ is denied authorization, the $\$ 50$ represents savings attributable to PA. The most elementary method of measuring the savings of PA is to add up denials and calculate their dollar value. It is important to note that whatever savings occur are offset, at least partially, by the cost of running the PA program.

Prior authorization also affects prescribing patterns in ways that either cut costs or increase them, and these effects should be included in the financial evaluation. As physicians and pharmacists learn the PA guidelines, drug selection decisions may change--even without a formal request (and denial) of an authorization. This cuts costs. PA may be cost-generating if more expensive or poorer quality products are used as substitutes for the products requiring PA, or if it causes needed care to not be delivered. This can result in reduced quality; consequently, additional services, drugs, or other medical services will be required. Prior authorization also may negatively affect patients if potentially beneficial drugs (e.g., drugs for acne) are not prescribed because of PA paperwork. The potential of PA to impair quality is attenuated somewhat if PA guidelines are based on the latest medical knowledge. 
Prior authorization and similar programs should operate in a way that does not unduly hinder patient care. A program that achieves its goal of altering prescribing, but does so at the expense of provider and patient satisfaction, may not be beneficial in the longer term in a competitive marketplace that emphasizes customer satisfaction. The way these programs operate is tied directly to patient and prescriber satisfaction. Long processing times for PA requests may delay the start of therapy and diminish quality of care. High denial rates may indicate poor guideline interpretation or provider communication, both of which are program administration problems. Thus, measurement of PA program performance along such dimensions as response times, approval rates, and access is important in assessing the degree to which the program is as efficient as it is effective.

\section{THE IOWA MEDICAID DRUG PA PROGRAM}

The lowa Medicaid drug program, which covers roughly 250,000 recipients and more than four million prescriptions, ${ }^{5}$ initiated PA in October 1992 and expanded it in August 1993. All guidelines for the PA program were developed by the Iowa Medicaid Drug Use Review Commission, a committee of prescribers and pharmacists that conducts the state's retrospective drug use review program and related activities. During the study, the program included prior authorization for 16 categories of individual medications, each with specific criteria. These broad categories included: amphetamine or ampheta- mine-like sympathomimetics for certain diagnoses; some legend and nonlegend multivitamins, tonic preparations, and associated combinations for specific conditions; clozapine under strict criteria; single-source NSAIDs; single-source benzodiazepine sedative hypnotics; $\mathrm{H}_{2}$-receptor antagonists and sucralfate at full therapeutic doses; omeprazole; misoprostol; growth hormones; prescription antiacne products; topical tretinoin; nonsedating antihistamines; dipyridamole; cephalexin hydrochloride monohydrate; epoetin; and filgrastim.

The fiscal agent in charge of administering the PA program used two full-time pharmacists and two customer representatives. Prior authorization requests were accepted by either telephone, facsimile, or mail. The program operated 51.5 hours per week, including limited weekend and evening hours.

\section{PROGRAM OPERATIONS METHODS}

One concern of any initiative such as PA is that it operate efficiently as well as produce savings and better outcomes. To measure efficiency, the lowa PA program was evaluated on workload, response time, and approval rates. Program operations data were recorded by PA personnel for every inquiry during a two-week period (August 16-August 29, 1995). Inquiries dealing with coverage decisions and medication requests were logged daily by PA personnel in a computer database (see Table 1). For this study, they also logged the time of day a new request was resolved. Extension requests (requests for patients to continue a PA medication) and all other

Table 2. Approval Rates by Therapeutic Categories and Type of Request

\begin{tabular}{|c|c|c|c|c|}
\hline \multirow{2}{*}{ CATEGORY } & \multicolumn{2}{|c|}{ NEW PAs } & \multicolumn{2}{|c|}{ EXTENSION PAS } \\
\hline & Number of Requests & Approval Rate & Number of Requests & Approval Rate \\
\hline Amphetamine products & 17 & $76.5 \%$ & 37 & $97.3 \%$ \\
\hline Antiacne products & 49 & 65.3 & 25 & 100.0 \\
\hline Antiulcer products & 190 & 85.8 & 185 & 88.1 \\
\hline Benzodiazepines & 28 & 78.6 & 48 & 95.8 \\
\hline Clozapine & 42 & 92.9 & - & - \\
\hline Dipyridamole & 2 & 100.0 & 3 & 100.0 \\
\hline Hematopoietic agents & 4 & 75.0 & 9 & 55.6 \\
\hline Nonsedating antihistamine products & 66 & 57.6 & 54 & 98.1 \\
\hline Nonsteroidal antiinflammatories (NSAIDs) & 100 & 63.0 & 36 & 100.0 \\
\hline Tretinoin & .23 & 52.2 & - & - \\
\hline Vitamin products & 11 & 81.8 & 2 & 100.0 \\
\hline TOTAL & 532 & $74.4 \%$ & 399 & $92.5 \%$ \\
\hline
\end{tabular}


activities were logged manually using daily log sheets (see Tables 2,3 , and 4). The investigators developed the data collection forms and methods of data collection with the collaboration of the fiscal agent. Directions for gathering information were presented both verbally and in writing to the PA personnel.

\section{DRUG USE AND EXPENDITURES METHODS}

In addition to operational performance, the PA program was assessed for effects on drug use and expenditures during the state's 1995 Fiscal Year (FY). The fiscal agent provided monthly prescription claims summaries that included utilization and price information. These data covered both a baseline period prior to the initiation of the PA program (up to two years for some drug classes) and the period from implementation of the PA program through the end of FY 1995. The fiscal agent also supplied data on the cost of operating the PA program for FY 1995 , listed by drug class or guideline. The analysis was limited to drug expenditures; it did not assess other medical (nondrug) expenses incurred as a consequence of PA guidelines. For example, an office visit may be generated each time a drug "promoted" by PA fails and an altemative is requested.

Analyses were performed separately for four major categories of PA guidelines. The savings were measured to reflect the effect of the PA guidelines on drug prescribing patterns. Rather than place a value on actual denials, program-wide utilization before PA was compared to program-wide utilization after PA. This approach was deemed appropriate for guidelines that encourage generic or therapeutic alternates as initial therapy, as well as those that encourage lower doses of therapy.

The process involved six steps. The first was to select an indicator that reflects the behavior(s) encouraged by the guideline. These indicators measured the changes in prescribing patterns associated with prior authorization. The indicators of prescribing behaviors for the four categories included:

- the proportion of all antiarthritic prescriptions which were for generic (vs. single-source) products;

- the proportion of all benzodiazepine prescriptions which were for generic (vs. single-source) products;

- the proportion of all antihistamine prescriptions that were for sedating (vs. nonsedating) products; and

- the proportion of antiulcer therapy days that were for lower maintenance doses (vs. higher therapeutic doses)

Second, baseline drug use before PA implementation was quantified and expressed in terms of drug use or prescribing patterns, based on numbers of prescriptions rather than monetary values. To determine baseline performance, data for 12 to 24 months (depending on category) before prior authorization were assessed.

Third, drug use during FY 1995 was quantified and compared to the baseline data. This step revealed the effect of PA on prescribing patterns or drug use.

Fourth, changes in performance or prescribing patterns were assigned monetary values, using prices paid by Medicaid during the study period (FY 1995). Prices were adjusted to reflect estimated rebates from drug manufacturers. Overall, the lowa Medicaid program received rebates amounting to about $20 \%$ of drug payments in 1995. Since the rebates differ for branded and generic products, the assumption was made that brand name products were associated with a $20 \%$ rebate and generic products were associated with no rebate. It also was assumed that nonsedating antihistamine and antiulcer products were branded and, hence, associated with rebates, while sedating antihistamines were assumed to be generic. Results were recalculated using a 30\% rebate for branded products and none for generics. This step addressed the question: What is the amount saved (or spent) in drug expenditures because PA has altered prescribing patterns?

The research design was a before/after comparison, without a control group. As always, the absence of a control group is problematic because other factors affecting program-wide drug use are not detected. An attempt was made to account for this uncertainty in the fifth step: sensitivity analysis. Also, the influence of factors other than PA on prescribing patterns was unknown, although no other initiatives aimed at altering prescribing were identified during the study period. Therefore, the sensitivity analysis arbitrarily assumed other factors might account for up to one-third of the changes in prescribing, and the savings were recalculated. The data for each category are shown in Table 5.

Up to this point, the analysis has measured and quantified the effects of PA on drug use and expenditures. The result is a gross savings in the drug budget. In the sixth and final step, the gross savings were reduced by the cost incurred in achieving them. The administrative cost (staffing and overhead) of running the entire PA program was $\$ 180,000$, according to the fiscal agent. Based on prescription claims and average time to resolve a PA request for each category, the assumption was made that $90 \%$ of the total program effort, or $\$ 162,000$, was devoted to the four categories. This was allocated by using the volume of new PAs during the two-week data collection period and the average number of minutes to process a request in each category. The latter figure was provided by the fiscal agent.

Multiplying the number of new requests by the appropriate processing time and summing over all types of guidelines provided a total PA effort. The percentage of effort devoted to each category was multiplied by the total cost to give the cost for that category (see Table 5).

\section{OPERATIONAL RESULTS}

During the two-week data collection period, 1,402 contacts were tracked. Of these, $92.6 \%$ were telephone contacts. An additional $6.2 \%$ were received by fax, and the remaining $1.2 \%$ of contacts arrived by mail. These contacts included new PA requests, requests for $P A$ extensions, and related calls ranging from general program questions to providers submitting additional patient information. The sample data appeared to be representative of the 
normal operations of the fiscal agent. When sample statistics were compared to totals provided by the fiscal agent, overall approval rates were comparable ( $82.9 \%$ for the sample, $83.8 \%$ reported for a 12-month period). The total number of new requests was slightly higher for the sample, averaging 266 new PA requests per week. Averaged totals for May, June, and July 1995 were 200 requests per week. This increase in August was consistent with higher seasonal rates in August of 1993 and 1994. Prior authorization request rates among medication classifications were also similar between the sample and those reported in previous months. For example, antihistamine requests for the sample were $13.1 \%$ of all new PA requests and $12.3 \%$ when totals for May, June, and July 1995 were averaged.

Table 1 depicts the volume of contacts for each day of the week. During the two-week study period, a total of 931 PA requests were received. Of these, 532 were new PA requests; 399 were requests for extensions. "Other Contacts" related to customer service and informational calls, and represented $31.7 \%$ of contacts between the fiscal agent and the patients and providers. Based on data from the two-week study period, the data depict a fairly consistent number of total contacts handled by the PA personnel during the work week. Even though weekend rates were for reduced hours, the per-hour averages were still below the weekday averages.

\section{Approval Rates}

Overall, $82.9 \%$ of the 931 new and extension PA requests were approved for coverage. Of the 532 new PA requests studied, $74.4 \%$ (396) were approved for coverage. Of the 136 denied,
$58.1 \%$ were due to lack of both previous trials and therapy failures with covered antihistamines, NSAIDs, and over-the-counter benzoyl peroxide products. The remaining denials were based on 13 different denial categories specific to the medication. Of the 399 requests for PA extensions, 92.5\% (369) were approved for further coverage.

A review across therapeutic categories revealed that the approval rates for new PA requests ranged from a low of $52.2 \%$ to a high of $100 \%$ (Table 2). This range narrowed $(57.6 \%$ to $92.9 \%$ approval) when including only those categories with at least 30 PA requests. For extensions, the range of approval was a low of $55.6 \%$ to a high of $100 \%$. Again, accounting for only those therapeutic categories with at least 30 requests for extension, the range narrowed ( $88.1 \%$ to $100 \%$ approval).

\section{Response Time}

Because of differences in procedures for extending an existing $P A$ and researching a new PA request, the response times were calculated separately. Also, response times by drug classification and by outcome (approval vs. denial) also were reviewed.

The average response time for new requests and extensions combined was 52.4 (s.d.=101.8) minutes. Viewed separately, new prior authorization response time averaged 73.2 (s.d. $=119.6$ ) minutes and extension response time averaged 28 ( $s . d .=67.5)$ minutes (see Tables 3 and 4).

When new PA requests were reviewed between medication categories, overall response times varied considerably. This was likely due to the variations in guidelines and the amount of research needed to verify that the procedures leading up to the PA

Table 3. Mean Response Times for New PA Requests (in minutes)

\begin{tabular}{l|c|c|c}
\hline Medication Category & Mean Approval Response (s.d.) & Mean Denial Response (s.d.) & Overall Mean Response (s.d.) \\
\hline Amphetamines & $115.3(254.9) \mathrm{n}=13$ & $39.5(23.3) \mathrm{n}=2$ & $105.2(237.6) \mathrm{n}=15$ \\
\hline Antiacne & $39.1(52.4) \mathrm{n}=32$ & $138.3(311.8) \mathrm{n}=17$ & $73.5(1.90 .9) \mathrm{n}=49$ \\
\hline Antihistamines & $56.8(62.7) \mathrm{n}=35$ & $59.3(64.4) \mathrm{n}=27$ & $57.9(62.9) \mathrm{n}=62$ \\
\hline Antiulcer & $86.0(84.0) \mathrm{n}=152$ & $35.2(56.1) \mathrm{n}=21$ & $79.8(82.7) \mathrm{n}=1.73$ \\
\hline Benzodiazepines & $54.1(87.4) \mathrm{n}=20$ & $49.7(47.5) \mathrm{n}=6$ & $53.0(79.1) \mathrm{n}=26$ \\
\hline Clozapine & $138.0(244.5) \mathrm{n}=37$ & $88.5(79.9) \mathrm{n}=2$ & $135.5(238.6) \mathrm{n}=39$ \\
\hline Dipyridamole & $200.0(130.1) \mathrm{n}=2$ & - & $200.0(130.1) \mathrm{n}=2$ \\
\hline Hematopoietics & $11.0 .0(140.9) \mathrm{n}=3$ & $9.0(N . A.) \mathrm{n}=1$ & $44.8(125.6) \mathrm{n}=4$ \\
\hline NSAIDs & $50.4(56.5) \mathrm{n}=60$ & $47.2(55.0) \mathrm{n}=35$ & $72.0(77.5) \mathrm{n}=23$ \\
\hline Tretinoin & $64.4(65.5) \mathrm{n}=12$ & $80.3(91.3) \mathrm{n}=1.1$ & $7.3(9.5) \mathrm{n}=9$ \\
\hline Vitamin agents & $5.1(5.5) \mathrm{n}=7$ & $15.0(19.8) \mathrm{n}=2$ & $73.2(119.6) \mathrm{n}=497$ \\
\hline Mean & $76.6(116.0) \mathrm{n}=373$ & $63.1(129.6) \mathrm{n}=124$ & \\
\hline$-n=0$ & & & \\
\hline
\end{tabular}


Table 4. Mean Response Times for Extension Requests by Therapeutic Category (in minutes)

\begin{tabular}{|c|c|c|c|}
\hline Medication Category & Mean Approval Response (s.d.) & Mean Denial Response (s.d.) & Overall Mean Response (s.d.) \\
\hline Amphetamines & $2.7(7.1) n=33$ & - & $2.7(7.1) \mathrm{n}=33$ \\
\hline Antiacne & $27.0(36.9) n=25$ & - & $27.0(36.9) n=25$ \\
\hline Antihistamines & $7.0(11.5) n=51$ & - & $7.0(11.5) \mathrm{n}=51$ \\
\hline Antiulcer & $47.7(56.9) \mathrm{n}=149$ & $30.3(54.4) n=22$ & $45.5(56.7) \mathrm{n}=171$ \\
\hline Benzodiazepines & $27.6(149.5) n=45$ & - & $27.6(149.5) \mathrm{n}=45$ \\
\hline Clozapine & - & - & - \\
\hline Dipyridamole & $8.0(13.9) n=3$ & - & $8.0(13.9) n=3$ \\
\hline Hematopoietics & $4.0(3.1) \mathrm{n}=5$ & $3.0(4.7) \mathrm{n}=4$ & $3.6(3.6) n=9$ \\
\hline NSAIDs & $5.4(13.3) \mathrm{n}=36$ & - & $5.4(13.3) n=36$ \\
\hline Tretinoin & - & - & - \\
\hline Vitamin agents & $56.0(13.3) \mathrm{n}=2$ & - & $56.0(13.3) \mathrm{n}=2$ \\
\hline Mean & $28.1(68.6) \mathrm{n}=349$ & $26.11(50.9) \mathrm{n}=26$ & $28.0(67.5) \mathrm{n}=375$ \\
\hline
\end{tabular}

$-n=0$

were followed. For instance, prior authorizations for vitamins simply required that specific diagnoses be provided. In contrast, agents such as clozapine required a specific diagnosis, plus verification of unsuccessful trials with three or more other antipsychotic medications, a score of at least 50 on the Brief Psychiatric Rating Scale, and that the patient was treatment-resistant. Thus, the additional requirements could demand more time for verification before an approval or denial could be provided

Tables 3 and 4 show the mean response times for new and extension PA requests, respectively, by drug classification. As would be hypothesized, response times for new PA requests were generally longer than those for extensions. Medication categories requiring the longest response time for a new request were clozapine (135.5 minutes), and amphetamine agents (105.2 minutes). Although standard deviations were large, no category had average response times greater than two and one-half hours.

\section{RESULTS}

\section{Drug Use and Expenditures}

A summary of the potential effects of PA on drug use and drug expenditures for the four categories is shown in Table 5. The changes in behavior or drug use between the baseline period and study period were quite pronounced. In 1995, generic prescriptions represented $87 \%$ and $89 \%$ of all antiarthritic and benzodiazepine prescriptions; during the baseline period, this figure ranged from $49 \%$ to $54 \%$ every month for both categories. Similarly, low-dose antiulcer days represented about $35 \%$ of all antiulcer days during the baseline period, and this figure was about $57 \%$ during the study period. Finally, the use of sedating antihistamines, ranging from $48 \%$ to $60 \%$ of all antihistamine prescriptions during the baseline months, was $91 \%$ in the study period. Given the differences in prices between the alternatives (i.e., a generic prescription compared to brand), the potential gross savings were: a) antiarthritics: $\$ 1,493,000$; b) benzodiazepines: $\$ 1,475,000 ; c)$ antiulcer: $\$ 738,000$; and d) antihistamines: $\$ 290,000$. As mentioned earlier, other factors beside PA may have affected prescribing patterns between the baseline and study periods; to account for this, an assumption was made that two-thirds of the change was due to PA and the remainder to other factors. Thus, the gross savings for all categories studied were estimated to range from $\$ 2,676,000$ to $\$ 3,996,000$. Also included in Table 5 is the estimated administrative cost associated with each category, totaling $\$ 162,000$. Thus, total net savings (gross savings less administrative costs) for the four categories was $\$ 2.51$ million to $\$ 3.83$ million. By category, the net savings ranges were: a) antiarthritics: $\$ 970,000$ to $\$ 1,463,000$; b) benzodiazepines: $\$ 982,000$ to $\$ 1,469,000$; c) antiulcer: $\$ 388,000$ to $\$ 632,000$; and d) antihistamines: $\$ 174,000$ to $\$ 270,000$.

\section{Limitations}

Since the research design is a before/after design, without a control group, program-wide utilization before PA is compared to program-wide utilization after PA. With the absence of a control group other factors affecting program-wide drug use are not detected. For instance, program-wide use of a particular drug may decline if enrollment (number of patients) declines; however, in the lowa Medicaid program, the number of drug 
recipients has been increasing." The movement to managed care would have a minimal effect on program-wide drug use, because in lowa Medicaid, the drug benefit is outside the managed care contract. A third possibility is changes in drug products and policies, such as the availability of a new generic or payment limits, that alter prescribing. This possibility was believed to be minimal during the study period.

Neither the operational nor the financial analysis measures long-term effects of the program and whether or not it affects patient health or satisfaction in either a positive or negative manner. As health care markets evolve and become more competitive, these may become important to measure. In assessing operational performance, the data collection period of two weeks was relatively short. A sampling plan that covered a longer time period would be more beneficial from a managerial point of view, and perhaps more efficient. This analysis viewed the PA program solely from the viewpoint of the third-party payor. The perceptions of prescribers, pharmacists, and patients as to the ease of administration of the PA program and its effect on quality of care are important and deserve monitoring. This analysis also relied exclusively on program-wide data. Assessing the PA program from the vantage point of individual patients would add perspective to the analysis presented here. The course of events for the patient following denial of a PA request is unknown. These questions cannot be answered with the level of data used in the analysis presented here.

\section{CONCLUSION}

Like other programs, PA also must be monitored with respect to operational performance. While performance can be measured similarly to claims processing, PA performance is more critical because it occurs in the midst of the service delivery process. Unnecessary delays or denials can adversely affect

Table 5. Drug Use, Savings, and Administrative Costs of Prior Authorization in FY 1995

\begin{tabular}{|c|c|c|c|c|}
\hline & Antiarthritcs & Benzodiazepines & Antihistamines & Antiulcer \\
\hline Baseline period & $10 / 90-9 / 92$ & $10 / 90-9 / 92$ & $11 / 91-7 / 93$ & $10 / 90-9 / 92$ \\
\hline Units used, baseline ${ }^{a}$ & 17,000 & 10,600 & 3,500 & 309,000 \\
\hline Units used, FY $95^{\mathrm{a}}$ & 17,800 & 10,900 & 3,000 & 369,000 \\
\hline \multicolumn{5}{|l|}{ Desired behavior } \\
\hline baseline $e^{\mathrm{b}}$ & $50.8-54.1$ & $48.8-54.3$ & $48-60$ & $33.6-35.7$ \\
\hline FY95 & 86.7 & 88.6 & 90.8 & 56.7 \\
\hline Potential influence of $\mathrm{PA}^{\mathrm{C}}$ & 72,100 & 49,300 & 11,200 & 983,400 \\
\hline Price difference, FY95 ${ }^{\mathrm{d}}$ & $\$ 20.71$ & $\$ 29.92$ & $\$ 25.93$ & $\$ 0.75$ \\
\hline Potential PA savings & $\$ 1,493,000$ & $\$ 1,475,000$ & $\$ 290,000$ & $\$ 738,000$ \\
\hline $\begin{array}{l}\text { Potential PA savings, } \\
\text { alternate rebate }\end{array}$ & $\$ 1,211,000$ & $\$ 1,245,000$ & $\$ 240,000$ & $\$ 649,000$ \\
\hline $\begin{array}{l}\text { Potential PA savings, } \\
\text { alternate effect of } \mathrm{PA}^{\mathrm{r}}\end{array}$ & $\$ 1,000,000$ & $\$ 988,000$ & $\$ 194,000$ & $\$ 494,000$ \\
\hline Costs to administer PA & $\$ 30,000$ & $\$ 6,000$ & $\$ 20,000$ & $\$ 106,000$ \\
\hline \multicolumn{5}{|c|}{ Use expressed as prescriptions per month, except antiulcer category which was days of therapy per month. } \\
\hline \multicolumn{5}{|c|}{$\begin{array}{l}\text { Range of monthly data during baseline period and annual data for FY95. For antiarthritics and benzodiazepines, the figure is "percent prescriptions, generic"; } \\
\text { for antihistamines, it is "percent prescriptions, sedating"; for antiulcer, it is "percent days, low dose." }\end{array}$} \\
\hline \multicolumn{5}{|c|}{ C Number of prescriptions (number of days for antiulcer) affected by change in behavior between FY95 and baseline. } \\
\hline \multicolumn{5}{|c|}{$\begin{array}{l}\text { d Difference in price between average brand and average generic prescriptions (or high and low dose days) in } 1.995 \text {, assuming } 20 \% \text { rebate on branded, non- } \\
\text { sedating, and all antiulcer products vs. no rebate on others. }\end{array}$} \\
\hline
\end{tabular}


patient outcomes. In the lowa Medicaid PA program, approximately $75 \%$ of new PA requests were approved; the average processing time for new requests was about 1.22 hours. As might be expected, response times and approval rates varied by type of guideline. While these operational performance statistics appear to be positive, they suffer from the lack of comparisons with other programs. Expected levels of performance in PA programs (turnaround times, approval rates, administrative costs) are not known, hence the difficulty in judging performance.

With respect to the financial consequences, the analysis focused on four prescribing behaviors encouraged by PA: prescribing generic antiarthritic and benzodiazepines before using branded products; prescribing sedating rather than nonsedating drugs and using antiulcer therapy in low or maintenance doses after a suitable time on high-dose therapy. Prior authorization, in these four categories, was associated with a net reduction in drug expenditures of $\$ 2.51$ million to $\$ 3.83$ million. This takes into account both the costs to run the program and rebates from drug manufacturers. To put this in perspective, this represented about $2 \%$ to $3 \%$ of drug vendor payments. ${ }^{5}$

Based on this analysis and other published works, prior authorization appears to have a marked effect on prescribing patterns. In the lowa program, for instance, two years after implementation of PA, the proportions of generic antiarthritic and benzodiazepine use were much higher than before PA ( $33 \%$ and $38 \%$, respectively); yet PA approval rates were $63 \%$ and $78 \%$, respectively. Prior authorization guidelines may have been responsible for changing prescribing behaviors, so that potentially deniable prescribing decisions were altered before formal requests were made. Whether such changes in behavior would be retained in the absence of the PA program is open to speculation, but given the half-life of other programs, the likelihood is that the effects of the PA program would diminish over time.

In conclusion, this article has presented a means of assessing the operational performance of a drug prior authorization program as well as a method of assessing its potential effect on prescribing and drug expenditures. The assessment, from the viewpoint of the third-party payor, uses program-wide data that are commonly available from drug plan administrators. By tracking a minimal number of variables during the daily operations of a prior authorization program, managers can easily assess both the financial savings and the efficiency of their programs. As these programs continue to come under fire by various parties within the health care system, quick and reliable measures of program quality will prove beneficial in supporting the use of these programs.

\section{$\Delta \quad$ References}

1. National Pharmaceutical Council and The-Lewin Group, Pharmaceutical benelits under state medical assistance programs, Reston, VA: National Pharmaceutical Council, 1996; 3-18.

2. Smalley, WE, Griffin, MR, Fought, RL, et al. Ef-

fect of a prior-authorization requirement on the use of nonsteroidal antiinflammatory drugs by medicaid patients. J Managed Care Pharm 1996 2(2): 158-164.

3. Smalley, WE, Griffin, MR, Fought, RL, Sullivan, $\mathrm{L}$ and Ray, WA. Effect of a Prior-Authorization Requirement on the Use of Nonsteroidal Antiinflammatory Drugs by Medicaid Patients. N Eng! J Med 332: 1612-17, 1995.
4. Kotzan, JA, McMillan, JA, Jankel, CA and Foster, AL. Initial Impact of a Medicaid Prior Authorization Program for NSAID Prescriptions. Journal of Research in Pharmaceutical Economics 5(1): 25 41, 1993

5. Wilford, BB Pharmaceutical Benefits Under State Medical Assistance Programs, Reston, VA: National Pharmaceutical Council, 1995; 319 